\title{
Topology of the Configuration Space of Polygons as a Codimension One Submanifold of a Torus
}

\author{
By
}

Takanori HinokUmA * and Hiroo SHIGA**

\begin{abstract}
We study the topology of polygons with fixed side length in Euclidean plane by Morse theory.
\end{abstract}

\section{$\S 1$. Introduction}

We study the space of oriented congruence classes of polygons with fixed side length in Euclidean plane $E^{2}$. Set

$$
X_{n+2}=\left\{\left(z_{1}, \cdots, z_{n+2}\right) \in \mathbb{C}^{n+2}: \sum_{i=1}^{n+2} z_{i}=0\right\}
$$

and

$$
N_{n+2}=X_{n+2}-\{(0, \cdots, 0)\} / \mathbb{C}^{*}=\left\{\left[z_{1}, \cdots, z_{n+2}\right] \in \mathbb{C} P^{n+1}: \sum_{i=1}^{n+2} z_{l}=0\right\},
$$

where $\mathbb{C}^{*}(=\mathbb{C}-\{0\})$ acts on $X$ diagonally. Then $N_{n+2}$ may be regarded as the space of oriented similarlity classes of $(n+2)$-gons in $E^{2}$.

For a positive number $r$, we set

$$
M_{n+2, r}=\left\{\left[z_{1}, \cdots, z_{n+2}\right] \in N_{n+2}:\left|z_{1}\right|=\left|z_{2}\right|=\cdots=\left|z_{n}\right|=\left|z_{n+2}\right| \neq 0,\left|z_{n+1}\right|=\eta\left|z_{n+2}\right|\right\} .
$$

Then by setting $\frac{z_{i}}{z_{n+2}}=w_{\imath}$, we have

Communicated by K. Saito, March 12, 1997. Revised January 6, 1998.

1991 Math. Subject Classification (s): 57N65, 58E05

* Department of Mathematics, College of Science, University of the Ryukyus, Nishihara, Okınawa 903-0129. Japan.

* * Department of Mathematics, College of Science, University of the Ryukyus, Nishihara. Okinawa 903-0129, Japan. 


$$
\begin{aligned}
M_{n+2, r} \simeq\left\{\left(w_{1}, w_{2}, \cdots, w_{n+1}, 1\right) \in\left(\mathbb{C}^{*}\right)^{n+1}: \begin{array}{l}
\left|w_{1}\right|=\cdots=\left|w_{n}\right|=1 \\
w_{n+1}=-\left(w_{1}+\cdots+w_{n}+1\right) \\
\left|w_{n+1}\right|=r
\end{array}\right\} \\
\simeq\left\{\left(w_{1}, w_{2}, \cdots, w_{n}\right) \in T^{n}:\left|w_{1}+\cdots+w_{n}+1\right|=r\right\}
\end{aligned}
$$

where $\simeq$ means diffeomorphic. Hence the space of similarlity classes of $(n+2)$ -gons whose $(n+1)$-sides have equal length may be regarded as a torus $T^{n}$, if we regard $(n+1)$-gons as a degenerate $(n+2)$-gons.

Set

$$
W_{n+2, r}=\left\{\left(w_{1}, \cdots, w_{n}\right) \in T^{n}:\left|w_{1}+\cdots+w_{n}+1\right| \geq r\right\},
$$

where $r$ is a nonnegative real number. $W_{n+2, r}$ is the configuration space of $(n+$ $2)$-gons whose $(n+1)$-side length are 1 and the other one is greater than or equal to $r$. We define a smooth function $f$ on $T^{n}$ as follows:

$$
f\left(e^{\imath x_{1}}, \cdots, e^{\imath x_{n}}\right)=-\left(\cos x_{1}+\cdots+\cos x_{n}+1\right)^{2}-\left(\sin x_{1}+\cdots+\sin x_{n}\right)^{2} .
$$

Then $f^{-1}(-1)=M_{n+2,1}$ may be regarded as the space of oriented congruence classes of $(n+2)$-gons whose length of $(n+2)$-sides are 1 . The critical points of $f: T^{n} \rightarrow \mathbb{R}$ are non degenerate except maximum. The value -1 is regular value if $n$ is odd and is critical value if $n$ is even. By using standard Morse theory for $f$, we have a handlebody decomposition of $W_{n+2, r}$. If $-r^{2}$ is a regular value of $f, M_{n+2, r}=\partial W_{n+2, r}$ is obtained from a sphere by succesive surgery. We give a cell structure of $T^{n+1}$ by the product complex of $S^{1}=e^{0} \cup e^{1}$.

By observing attaching maps of handlebodies, we get the following results.

Theorem 1. For $0<r \leq n+1$, the space $W_{n+2, r}$ is homotopy equivalent to $\left(T^{n+1}\right)^{k}$, the $k$-skelton of $T^{n+1}$, where $k=\left[\frac{n+1-r}{2}\right]$. If $r \leq 0, W_{n+2, r}$ is the whole space $T^{n}$ and $W_{n+2, r}=\emptyset$ if $r>n+1$

By using Theorem 1 and Lefschetz duality, we are able to know that the relative homotopy groups, $\pi_{\imath}\left(W_{n+2, r} M_{n+2, r}\right)=0$ for $i \leq n-k-1$, where $k$ is the integer such that $n-2 k-1<r \leq n-2 k+1$. Then we have the following results on the fundamental group of $M_{n, r}$ for $n \geq 4$.

Corollary 2. Let $n \geq 4$. Then

$$
\pi_{1}\left(M_{n+2, r}\right) \simeq \begin{cases}\mathbb{Z}^{n+1} & \text { if } 0 \leq r \leq n-3 \\ F_{n+1} & \text { if } n-3<r \leq n-1 \\ \{e\} & \text { if } n-1<r \leq n+1 .\end{cases}
$$

In case $n=3$, the inclusion $i: M_{5,1} \rightarrow W_{5,1} \simeq\left(T^{4}\right)^{1}$ induces surjection (but not 
isomorphism) on the fundamental groups.

We can also calculate the homology group of $M_{n+2, r}$ easily by Theorem 1 . Since manifold $M_{n+2, r}\left(-r^{2}\right.$ is regular value of $\left.f\right)$ is closed orientable codimension 1 submanifold of $T^{n}$ which bounds $W_{n, r}$ it is a $\pi$-manifold and oriented cobordant to zero. The manifold $M_{n+2, r}$ is obtained from a sphere by successive surgeries, in particular we have the following from table I (in Section 2) :

Proposition 3. For $n \geq 3$, we have

$$
\begin{aligned}
& M_{n+2, r} \simeq S^{n-1} \text { if } n-1<r<n+1 \\
& M_{n+2, r} \underset{n+1}{\#}\left(S^{1} \times S^{n-2}\right) \text { if } n-3<r<n-1,
\end{aligned}
$$

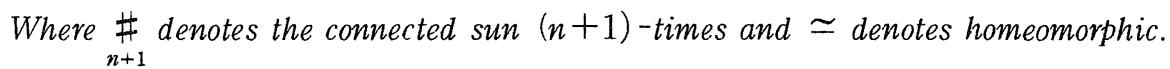

In case $n=4$, more general information was obtained by M. Kapovich and J. Milson ( $[\mathrm{K}-\mathrm{M}]$ Theorem 3). In particular, we have $M_{5,1}$ is diffeomorphic to $\sum_{4}$, the closed orientable surface of genus 4 . ([T-W], [Hav]). The homology group of $M_{n+2,1}$ is also calculated in [K-T-T]

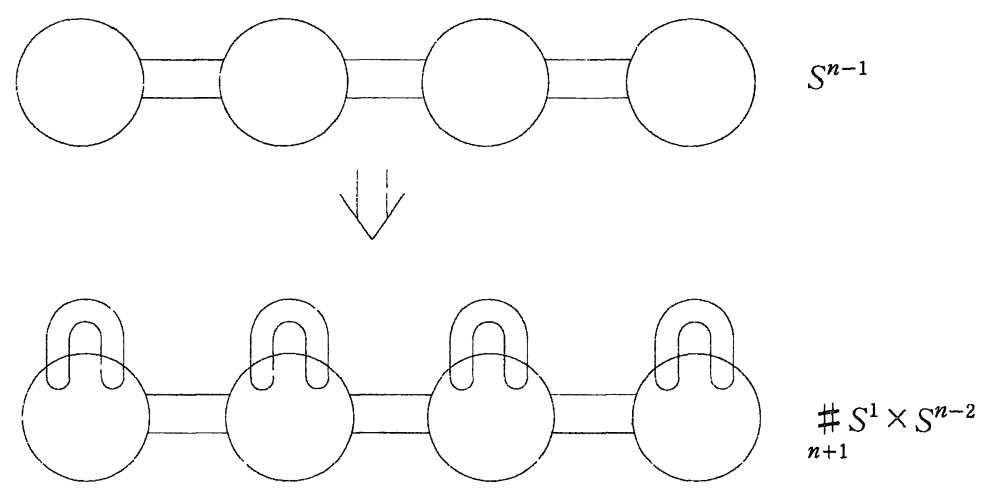

\section{§ 2. Critical Points of $f$}

We study the critical points of the function

$$
f\left(e^{i x_{1}}, \cdots, e^{i x_{n}}\right)=-\left(\cos x_{1}+\cdots+\cos x_{n}+1\right)^{2}-\left(\sin x_{1}+\cdots+\sin x_{n}\right)^{2}
$$

on $T^{n}$. Then 


$$
\frac{\partial f}{\partial x_{1}}=2\left(\cos x_{1}+\cdots+\cos x_{n}+1\right) \sin x_{i}-2\left(\sin x_{1}+\cdots+\sin x_{n}\right) \cos x_{\imath}
$$

and

$$
\sum_{i=1}^{n} \frac{\partial f}{\partial x_{i}}=\sum_{i=1}^{n} \sin x_{i}
$$

Hence the set of critical points of $f$ are the union of

$$
A=\left\{\left(e^{i x_{1}}, \cdots, e^{i x_{n}}\right) \in T^{n}: \sin x_{1}=\cdots=\sin x_{n}=0\right\}
$$

and

$$
B=\left\{\left(e^{\imath x_{1}}, \cdots, e^{\imath x_{n}}\right) \in T^{n}: \sum_{i=1}^{n} \cos x_{i}=-1 \text { and } \sum_{i=1}^{n} \sin x_{i}=0\right\} .
$$

On the set $B, f$ attains the maximum value 0 and $B$ is homeomorphic to $M_{n+1,1} \times S^{1}$. Set

$$
S_{k}=\left\{\left(\varepsilon_{1}, \cdots, \varepsilon_{n}\right) \in T^{n}: \varepsilon_{l}=1 \text { or }-1 \text { and the cardinality of }-1 \text { is } k .\right\} .
$$

Then

$$
A \cap B^{c} \subset \bigcup_{k=0}^{n} S_{k} \text { and } A \cup B \supset \bigcup_{k=0}^{n} S_{k}
$$

Lemma 2.1. At the critical point $x_{0}=(\underbrace{-1, \cdots,-1}_{k}, \underbrace{1, \cdots, 1}_{n-k})(k=0,1, \cdots$, $n$ ), the characteristic polynomial of the Hessian (of $f$ ) is :

$$
(\lambda-2 b)^{n-k-1}(\lambda+2 b)^{k-1}\left(\lambda^{2}+2 n \lambda-4 b\right),
$$

where $b=n-2 k+1$. Therefore the index of the critical point $x_{0}$ is $k$ if $b>0$ and $n$ $-k$ if $b<0$. (Note that in the case that $b=0$ f attains the maximum value 0 )

Proof. Since

$$
\frac{\partial^{2} f}{\partial x_{\imath} \partial x_{j}}=\left\{\begin{array}{l}
-2 \cos \left(x_{i}-x_{\jmath}\right) \\
-2+2(C+1) \cos x_{\imath}-2 S \sin x_{\imath}
\end{array}\right.
$$

where $C=\cos x_{1}+\cdots+\cos x_{n}$ and $S=\sin x_{1}+\cdots+\sin x_{n}$ at the point $x_{0}=$ $(\underbrace{-1, \cdots,-1}_{k}, \underbrace{1, \cdots, 1}_{n-k})$, 


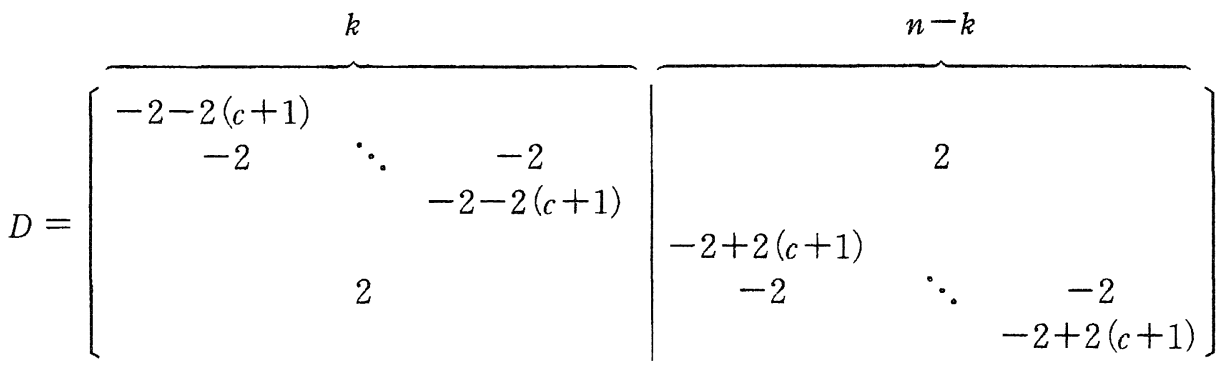

$$
\begin{aligned}
& =2\left[\begin{array}{ccc|ccc}
-1-(c+1) & & & & \\
-1 & \ddots & -1 & & 1 & \\
& & -1-(c+1) & & & \\
& 1 & & -1+(c+1) & & -1 \\
& & & & \ddots & -1+(c+1)
\end{array}\right] \\
& =2 D_{1}
\end{aligned}
$$

where $c=n-2 k$. We calculate the characteristic polynomial of $D_{1}$ instead of $D$ for simlicity.

$$
\left|\lambda I-D_{1}\right|=\left|\begin{array}{cccccc}
\lambda+1+b & & & & & \\
1 & \ddots & 1 & & -1 & \\
& & \lambda+1+b & & & \\
& -1 & & \lambda+1-b & & 1 \\
& & & & \ddots & \lambda+1-b
\end{array}\right|
$$

where $b=c+1=n-2 k+1$. We substruct the first column from the $i$-th $(i=1$, $\cdots, k)$ and add it to the $j$-th $(j=k+1, \cdots, n)$. Our determinant is equal to

$$
\left|\begin{array}{ccccccc}
\lambda+1+b & -\lambda-b & \ldots & -\lambda-b & \lambda+b & \ldots & \lambda+b \\
1 & \lambda+b & & & & & \\
\vdots & 0 & \ddots & 0 & & 0 & \\
1 & & & \lambda+b & & & \\
-1 & & & & \lambda-b & & \\
\vdots & & 0 & & 0 & \ddots & 0 \\
-1 & & & & & & \lambda-b
\end{array}\right|
$$

We then add the rows of from the second to the $k$-th to the first row and add $\frac{\lambda+b}{\lambda-b}$ times the rows of from the $k+1$-th to $n$-th to the first row also. This yields 


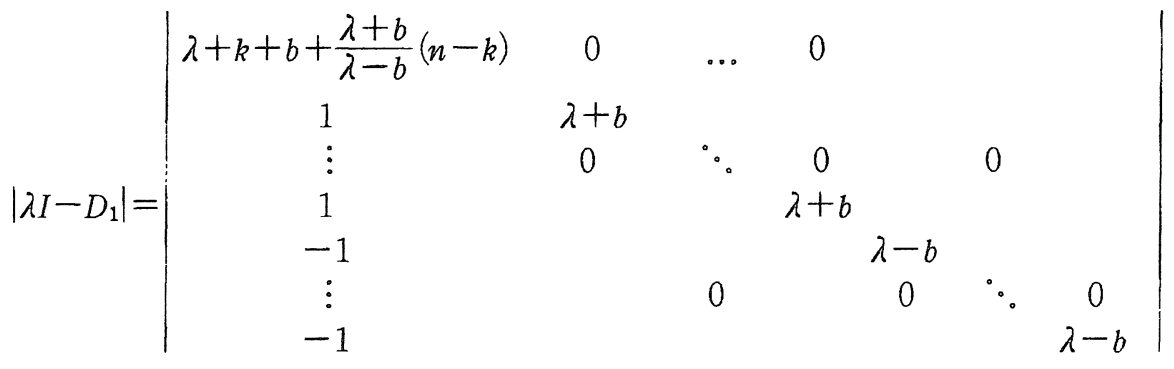

$$
\begin{aligned}
& =\left(\lambda+k+b+\frac{\lambda+b}{\lambda-b}(n-k)(\lambda+b)^{k-1}(\lambda-b)^{n-k}\right. \\
& =(\lambda+b)^{k-1}(\lambda-b)^{n-k-1}\left(\lambda^{2}+n \lambda-b^{2}+(n-2 k) b\right) \\
& =(\lambda+b)^{k-1}(\lambda-b)^{n-k-1}\left(\lambda^{2}+n \lambda-b\right)
\end{aligned}
$$

where the last equality is obtained by using $b=n-k+1$. Therefore the characteristic polynomial of $D\left(=2 D_{1}\right)$ is :

$$
(\lambda-2 b)^{n-k-1}(\lambda+2 b)^{k-1}\left(\lambda^{2}+2 n \lambda-4 b\right) .
$$

By Lemma 2.1, we have the following table:

\begin{tabular}{|c|c|c|}
\hline Critical value & index & the number of critical points \\
\hline$-(n+1)^{2}$ & 0 & 1 \\
\hline$-(n-1)^{2}$ & 1 & ${ }_{n+1} C_{1}$ \\
\hline$-(n-3)^{2}$ & 2 & ${ }_{n+1} C_{2}$ \\
\hline$\vdots$ & $\vdots$ & $\vdots$ \\
\hline$-(n-2 \mathrm{k}+1)^{2}$ & $k$ & ${ }_{n+1} C_{k}$ \\
\hline
\end{tabular}

table I

The number of critical points of index $k$ is the cardinality of the union of $S_{k}$ and $S_{n-k+1}$.

We denote the binomial coefficients ${ }_{n+1} C_{k}=\left(\begin{array}{c}n+1 \\ k\end{array}\right)$ by $P(k)$.

\section{§3. The Homotopy Type of $W_{n+2, r}$}

In this section, we study the homotopy type of

$$
W_{n+2, r}=\left\{\left(e^{\imath x_{1}}, \cdots, e^{\imath x_{n}}\right) \in T^{n}: f \leq-r^{2}\right\} .
$$

By Morse theory and Table I, $W_{n+2, r}$ is obtained from $W_{n+2, r+2}$ by attaching $P(k)$ handle bodies of $k$-dimension.

Let $n-3<r<n-1$ then $W_{r}$ is homotopy equivalent to $D^{n}\left(\simeq W_{n+2, r+2}\right)$ with $P(1)(=n+1) 1$-cells attached. Hence $W_{n+2, r}$ is homotopy equivalent to $n+$ 
1 times wedge of $S^{1}$, which is homotopy equivalent to 1 -skelton of $T^{n+1} \cdot W_{n+2, n-1}$ is also homotopy equivalent to $\left(T^{n+1}\right)^{1}$. (See Milnor $[\mathrm{M}: 1]$ Remark 3.4., p.20)

In order to study the case $n-5<r \leq n-3$, we must observe the attaching maps of 2 -cells. We examine at the critical point $P=(-1,-1,1, \cdots, 1)$ of index 2 . We can treat similarly at other critical points. Set

$$
T^{2}=\left\{\left(e^{i x_{1}}, e^{i x_{2}}, 1, \cdots, 1\right) \in T^{n}: x_{1}, x_{2} \in \mathbb{R}\right\}
$$

and let $f_{2}$ be the restriction of $f$ on $T^{2}$. Then $f_{2}$ attains maximum value $-(n-3)^{2}$ at $P$. For a small positive number $\varepsilon$, we set

$$
D_{\varepsilon}^{2}=\left\{f_{2} \geq-(n-3+\varepsilon)^{2}\right\} \subset T^{2} .
$$

Then $D_{\varepsilon}^{2}$ is diffeomorphic to a closed 2-dimensional disk containing $P$ as an interior point. We show that the attaching sphere of the 2-cell at $P$ may be regarded as $\partial D_{\varepsilon}^{2}$.

Around the critical point $P$, there is a local coordinate $\left(y_{1}, \cdots, y_{n}\right)$ of $T^{n}$ with $P=(0, \cdots, 0)$ such that $f$ is expressed as

$$
-y_{1}^{2}-y_{2}^{2}+y_{3}^{2}+\cdots+y_{n}^{2}-(n-3)^{2} \text {. }
$$

The attaching sphere $S_{L}^{1}$ of a 2 -cell in $W_{n+2, r}(n-3<r<n-1)$ can be regarden as

$$
\left\{\left(y_{1}, \cdots, y_{n}\right): y_{1}^{2}+y_{2}^{2}=\delta^{2}, y_{3}=\cdots=y_{n}=0\right\}
$$

$($ See $[\mathrm{Mi} 2])$. Set

$$
C=\left\{\left(y_{1}, \cdots, y_{n}\right) \in \mathbb{R}^{n}:-y_{1}^{2}-y_{2}^{2}+y_{3}^{2}+\cdots+y_{n}^{2} \geq 0\right\} .
$$

Then we see

$$
\partial D_{\varepsilon}^{2} \subset \mathbb{R}^{n}-C
$$

The sphere $S_{L}^{1}$ and $\partial D_{\varepsilon}^{2}$ both generate the group $\pi_{1}\left(\mathbb{R}^{n}-C\right) \simeq \pi_{1}\left(\mathbb{R}^{2}-\{(0,0)\}\right)$ $\simeq \mathbb{Z}$. Hence the two inclusions

$$
\begin{aligned}
& i_{1}: S_{L}^{1} \rightarrow \mathbb{R}^{n}-C \text { and } \\
& i_{2}: \partial D_{\varepsilon}^{2} \rightarrow \mathbb{R}^{n}-C
\end{aligned}
$$

are homotopic. Hence we have the following.

Lemma 3.1. The attaching map of 2-cell at $P$ is (free) homotopic to the composition of inclusion

$$
\partial D_{\varepsilon}^{2} \stackrel{\imath 1}{\rightarrow} T^{2}-\stackrel{\circ}{D}_{\varepsilon}^{2} \stackrel{\imath 2}{\longrightarrow} W_{n+2, n-3+\varepsilon}
$$


for some small positive number $\varepsilon$.

Set

$$
S_{j}=\left\{\left(1,1, \cdots, e^{i x}, 1, \cdots, 1\right) \in T^{n}: x_{i} \in \mathbb{R}\right\} \subset W_{n+2, n-1}(j=1, \cdots, n)
$$

and

$$
S_{n+1}=\left\{\left(e^{i x}, \cdots, e^{i x}\right) \in T^{n}: x \in \mathbb{R}\right\} \subset W_{n+2, n-1} .
$$

Since $W_{n+2, n-1}$ is homotopy equivalent to $D^{n}$ with $n+11$-cells attached and each 1 -cell can be regarded as the arc of $S$, containing the critical point of index 1 , $W_{n+2, n-1}$ is homotopy equivalent to the wedge of $S^{1}$

$$
S_{1} \vee \cdots \vee S_{n+1}
$$

Hence $\pi_{1}\left(W_{n+2, n-1}\right)$ is isomorphic to the free group $F\left(\alpha_{1}, \cdots, \alpha_{n+1}\right)$ of rank $n+1$. where $\alpha_{j}(j=1, \cdots, n+1)$ is represented by $S_{j}$. Let $\beta_{j}(j=1, \cdots, n)$ be the element of $\pi_{1}\left(T^{n}\right)$ represented by $S_{j}$, Then the inclusion $i: W_{n+2, n-1} \rightarrow T^{n}$ induces a homomorphism

$$
i_{\#}: \pi_{1}\left(W_{n+2, n-1}\right) \rightarrow \pi_{1}\left(T^{n}\right)
$$

such that $i_{\#}\left(\alpha_{j}\right)=\beta_{j}$ and $i_{\#}\left(\alpha_{n+1}\right)=\sum_{j=1}^{n} \beta_{j}$. By Lemma 3.1, the attaching map of 2 -cell at the critial point $P=(-1,-1,1, \cdots, 1)$ is the composition

$$
\partial D_{\varepsilon}^{2} \subset T^{2}-\stackrel{\circ}{D}_{\varepsilon}^{2} \stackrel{j}{\subset} W_{n+2, n-3+\varepsilon}\left(-W_{n+2, n-1}\right) .
$$

The inclusion $j$ induces homomorphism

$$
\begin{gathered}
\pi_{1}\left(T^{2}-\stackrel{\circ}{D}_{\varepsilon}^{2}\right) \stackrel{j_{\#}}{\longrightarrow} \pi_{1}\left(W_{n+2, n-3+\varepsilon}^{r}\right) \\
F\left(\gamma_{1}, \gamma_{2}\right) \stackrel{\|}{\longrightarrow} F\left(\alpha_{1}, \cdots, \alpha_{n+1}\right)
\end{gathered}
$$

such that $j_{\#}\left(\gamma_{1}\right)=\alpha_{1}$ and $j_{\#}\left(\gamma_{2}\right)=\alpha_{2}$, where $\gamma_{j}(j=1,2)$ is represented by $S_{j} \subset T^{2}$. Since the inclusion $\partial D_{\varepsilon}^{2} \rightarrow T^{2}-\stackrel{\circ}{D}_{\varepsilon}^{2}$ represents the commutater $\gamma_{1} \gamma_{2} \gamma_{1}^{-1} \gamma_{2}^{-1}$ in the fundamental group, its image in $\left(T^{n+1}\right)^{2}$ is trivial. The manifold $W_{n+2, n-3+\varepsilon}$ is homotopy equivalent to $\left(T^{n+1}\right)^{1}$ and it has the same homotopy type as $U_{P(2)}\left(T^{2}\right.$ $\left.-\stackrel{\circ}{D}_{\varepsilon}^{2}\right)\left(\subset T^{n+1}\right)$. Since $\left(T^{n+1}\right)^{2}=\cup_{P(2)} T^{2} \quad\left(\subset T^{n+1}\right)$ the homotopy equivalence $h_{1}: W_{n+2, n-3+\varepsilon} \rightarrow\left(T^{n+1}\right)^{1}$ can be extended to a map

$$
h_{2}: W_{n+2, n-3-\varepsilon} \longrightarrow\left(T^{n+1}\right)^{2}
$$


such that the induced map

$$
h_{2 *}: \pi_{*}\left(W_{n+2, n-3-\varepsilon}, W_{n+2, n-3+\varepsilon}\right) \longrightarrow \pi_{*}\left(\left(T^{n+1}\right)^{2},\left(T^{n+1}\right)^{1}\right)
$$

is isomorphic (both groups are isomorphic to $\mathbf{Z}^{P(2)}$ ). Then from the long exact sequence of homotopy groups and five Lemma, we obtain that $h_{2}$ is homotopy equivalence. We continue the above arguments for $n-2 k-1<r \leq n-2 k+1$ $(k \geq 3)$.

Proposition 3.2. Let $r<n-5$ and assume that there is a homotopy equivalence

$$
h_{r+2}: W_{n+2, r+2} \rightarrow\left(T^{n+1}\right)^{k-1},
$$

where $k$ is the integer such that $n-2 k-1<r \leq n-2 k+1$. (Note that $k \geq 3$.) Then $h_{r+2}$ can be extended to a homotopy equivalence

$$
h_{r}: W_{n+2, r} \rightarrow\left(T^{n+1}\right)^{k} .
$$

Proof. By Morse theory $W_{n+2, r}$ is homotopy equivalent to $W_{n+2, r+2}$ with $P(k)$ cells of dimension $k$ attached. We consider at the critical point $P_{k}=$ $(\underbrace{-1, \cdots,-1}_{k}, \underbrace{1, \cdots, 1}_{n-k})$ of index $k$. Set

$$
T^{k}=\left\{\left(e^{i x_{1}}, \cdots, e^{i x_{k}}, 1, \cdots, 1\right): x_{1}, \cdots, x_{k} \in \mathbb{R}\right\}
$$

and let $f_{k}$ be the restriction of $f$ on $T^{k}$. For a small positive number $\varepsilon$. we set

$$
D_{\varepsilon}^{k}=\left\{f_{k} \geq-(n-2 k+1+\varepsilon)^{2}\right\} .
$$

As in the previous case, the inclusion

$$
\partial D_{\varepsilon}^{k} \subset T^{\mathrm{k}}-\stackrel{\circ}{D}_{\varepsilon}^{k} \subset W_{n+2, n-2 k+1+\varepsilon}\left(\simeq\left(T^{n+1}\right)^{k-1}\right)
$$

is homotopic to the attaching map at $P_{k}$. Since $\pi_{k-1}\left(\left(T^{n+1}\right)^{k}\right) \simeq \pi_{k-1}\left(T^{n+1}\right)=0$, for $k \geq 3$. Hence we have an extension of $h_{r+2}$

$$
h_{r}: W_{n+2, n-2 k+1+\varepsilon} \cup\left(\bigcup_{i=1}^{P(k)} e_{i}^{k}\right) \rightarrow\left(T^{n+1}\right)^{k}
$$

such that $h_{r}$ induces isomorphisms on the relative homotopy groups

$$
h_{r *}: \pi_{*}\left(W_{n+2, n-2 k+1+\varepsilon} \cup\left(\bigcup_{i=1}^{P(k)} e_{i}^{k}\right) \rightarrow\left(T^{n+1}\right)^{k} .\right.
$$

such that $h_{r}$ induces isomorphisms on the relative homotopy groups

$$
h_{r *}: \pi_{*}\left(W_{n+2, n-2 k+1}, W_{n+2, n-2 k+1+\varepsilon}^{r}\right) \rightarrow \pi_{*}\left(\left(T^{n+1}\right)^{k},\left(T^{n+1}\right)^{k-1}\right) .
$$


Hence $h_{r}$ is homotopy equivalece by Whitehead Theorem.

Then by Proposition 3.2, proof of Theorem 1 is completed.

\section{$\S 4$. Topology of $M_{n+2, r}$}

By Theorem 1, we can compute the homology group of $M_{n+2, r}$. For $0<r$ $\leq n+1$, we choose an integer $k$ such that $n-2 k-1<r \leq n-2 k+1$. By Theorem $1, H\left(W_{n+2, r}, \mathbb{Z}\right)=0$ for $j \geq k+1$. We assume $n-2 k-1<r<n-2 k$ +1 . Then $W_{n+2, r}$ and $M_{n+2, r}$ are smooth compact connected manifolds such that $\partial W_{n+2, r}=M_{n+2, r}$. By Lefschetz duality and Theorem 1,

$$
H_{i}\left(W_{n+2, r}, M_{n+2, r}\right) \simeq H^{n-i}\left(W_{n+2, r}\right)=0
$$

for $i \leq n-k-1$. When $r=n-2 k+1, M_{n+2, r}$ has singular points and it is homotopy equivalent to

$$
M_{n+2, n-2 k+1+\varepsilon} \times I \cup\left(\bigcup_{j=1}^{P(k)} e_{j}^{k}\right)
$$

where $I$ is the closed interval $[0,1], e_{j}^{k}(j=1, \cdots, P(k))$ are $k$-cell and $\varepsilon$ is a small positive number. Hence by excision, we have

$$
\begin{aligned}
& H_{l}\left(W_{n+2, n-2 k+1}, M_{n+2, n-2 k+1}\right) \\
& \quad \simeq H_{l}\left(W_{n+2, n-2 k+1+\varepsilon} \cup M_{n+2, n-2 k+1+\varepsilon} \times I \cup\left(\bigcup_{j}^{k}\right), M_{n+2, n-2 k+1+\varepsilon} \times I \cup\left(\bigcup_{j} e_{j}^{k}\right)\right) \\
& \quad \simeq H_{l}\left(W_{n+2, n-2 k+1+\varepsilon}, M_{n+2, n-2 k+1+\varepsilon}\right) \\
& \quad=0 \text { if } i \leq n-k+1 .
\end{aligned}
$$

Proposition 4.1. Let $n$ be odd. Then $M_{n+2,1}$ is an $n-1$ dimensional connected closed manifold whose homology is as follows:

$$
\begin{array}{ll}
\text { (1) } H_{i}\left(M_{n+2,1}, \mathbb{Z}\right) \simeq \mathbb{Z}^{P(\imath)} & \text { for } 0 \leq i<\frac{n-1}{2} \\
\text { (2) } H_{i}\left(M_{n+2,1}, \mathbb{Z}\right) \simeq \mathbb{Z}^{2 P(i)} & \text { for } i=\frac{n-1}{2} \\
\text { (3) } H_{\imath}\left(M_{n+2,1}, \mathbb{Z}\right)=0 & \text { for } i>n-1 .
\end{array}
$$

Proof. By Theorem I, we have $W_{n+2,1} \simeq\left(T^{n+1}\right)^{\frac{n-1}{2}}$. From the long homology exact sequence of the pair $\left(W_{n+2,1}^{r}, M_{n+2,1}\right)$ and Poincaré duality we have (1). From the exact sequence 


$$
\begin{array}{ccc}
H & H \frac{n+1}{2}\left(W_{n+2,1}\right) \rightarrow H \frac{n+1}{2}\left(W_{n+2,1}, M_{n+2,1}\right) \rightarrow H \frac{n-1}{2}\left(M_{n+2,1}\right) & \rightarrow H \frac{n-1}{2}\left(W_{n+2,1}\right) \rightarrow 0 \\
\text { If Lefschetz dual } & \text { If L } \\
H \frac{n+1}{2}\left(\left(T^{n+1}\right)^{\frac{n-1}{2}}\right) & H \frac{n-1}{2}\left(W_{n+2,1}\right) & \mathbf{Z}^{P\left(\frac{n-1}{2}\right)}
\end{array}
$$

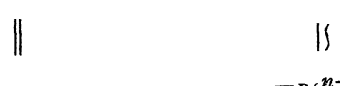

$0 \quad \mathbb{Z}^{P\left(\frac{n-1}{2}\right)}$

we have (2). By the dimensional reason, we have (3).

Proposition 4.2. Let $n$ be even. Then
(1) $\quad H_{i}\left(M_{n+2,1}, \mathbb{Z}\right) \simeq \mathbb{Z}^{P(\imath)}$
for $0 \leq i \leq \frac{n}{2}$
(2) $\quad H_{i}\left(M_{n+2,1}, \mathbb{Z}\right) \simeq \mathbb{Z}^{P(n-1-1)}$
for $\frac{n}{2}<i \leq n-1$
(3) $\quad H_{i}\left(M_{n+2,1}, \mathbb{Z}\right)=0$
for $i>n-1$.

Proof. By (4.3), $H_{i}\left(W_{n+2,1}, M_{n+2,1}\right)=0$ for $i \leq \frac{n}{2}$. Then from the long homology exact sequence, we have (1). From (4.2) and Lefschetz duality, $H_{\imath+1}\left(W_{n+2,1}, M_{n+2,1}\right) \simeq H_{\imath+1}\left(W_{n+2,1+\varepsilon}, M_{n+2,1+\varepsilon}\right) \simeq H^{n-\imath-1}\left(W_{n+2,1+\varepsilon}\right) \simeq H^{n-\imath-1}\left(\left(T^{n+1}\right)^{\frac{n}{2}-1}\right)$. For $i \geq \frac{n}{2}$, from the exact sequence,

$$
\begin{array}{cc}
\rightarrow H_{l+1}\left(W_{n+2,1}\right) \rightarrow & H_{l+1}\left(W_{n+2,1}, M_{n+2,1}\right) \rightarrow H_{\imath}\left(M_{n+2,1}\right) \rightarrow 0 \\
\| & \text { || } \\
& H_{n-i-1}\left(\left(T^{n+1}\right)^{\frac{n}{2}-1}\right) \\
& \mathbb{Q}^{P(n-1-1)}
\end{array}
$$

we have (2). (3) is dimensional reason.

By (4.1) and (4.2), we have $\pi_{1}\left(W_{n+2,1}, M_{n+2,1}\right)=0$ for $i \leq \frac{n-1}{2}$ if $n$ is odd and $\pi_{\imath}\left(W_{n+2,1}, M_{n+2,1}\right)=0$ for $i \leq \frac{n}{2}$ if $n$ is even. 
Corollary 2. Let $n$ be odd and $n \geq 3$. Then

$$
\pi_{i}\left(M_{n+2,1}\right) \simeq \pi_{i}\left(\left(T^{n+1}\right)^{\frac{n-1}{2}}\right) \quad \text { if } i \leq \frac{n-3}{2} .
$$

Let $n$ even and $n \geq 4$. Then

$$
\pi_{i}\left(M_{n+2,1}\right) \simeq \pi_{i}\left(\left(T^{n+1}\right)^{\frac{n}{2}-1}\right) \quad \text { if } i \leq \frac{n}{2}
$$

In particular,

Corollary 4.3.

$$
\pi_{i}\left(M_{n+2,1}\right) \simeq \mathbb{Z}^{P(1)} \quad \text { if } n \geq 4
$$

Example.From (4.2), $M_{n+2, n-1}$ is homotopy equivalent to

$$
S^{n-1} \vee \underbrace{S^{1} \vee \cdots \vee S^{1}}_{P(1)=n+1 \text { times }}
$$

\section{References}

[Hai] Hausmann, J. C., Sur la topologie des bras articulés, Lecturc Note in Math., 1474 (1990), 146-159.

[Hav] Havel, T.. The use of distances as a coordinates in computer-aided proofs of Theorems in Euclidean geometry, J. Symbolic Comput., 11 (1991), 579-593.

[K-M] Kapovich, M. and Milson, J., On the Moduli space of Polygons in the Euclidean Plane, $J$. Diff. Geo., 42 (1995), 133-164.

[K-T-T] Kamiyama, Y.. Tezuka, M. and Toma, T., The homology of equilatral polygon linkage, preprint.

[Mil] Milnor, J., Morse Theory. Ann. of Math. Stud., $\mathbf{5} \mathbb{1}$ (1963).

[Mi2] Lecture on the h-cobordism theorem, Princeton Univ. Press, 1965.

[S] Schoenberg I., Linkages and distance geometry I linkage, Indag. Math., 31 (1969), 42-52.

[T-W] Thurston, W., and Weeks. J., The mathematics of the three dimensional manifolds, Sci. Amer., 251 (1984) . 94-106 